\title{
Multi-view Multi-instance Multi-label Active Learning
}

\author{
Guoxian Yu, Yuying Xing, Jun Wang, Carlotta Domeniconi, Xiangliang Zhang
}

\begin{abstract}
Multi-view multi-instance multi-label learning (M3L) is a framework for modeling complex objects. In this framework, each object (or bag) contains one or more instances, is represented with different feature views, and simultaneously annotated with a set of non-exclusive semantic labels. Given the multiplicity of the studied objects, traditional M3L methods generally demand a large number of labeled bags to train a predictive model to annotate bags (or instances) with semantic labels. However, annotating sufficient bags is very expensive and often impractical. In this paper, we present an Active learning based M3L approach (M3AL) to reduce the labeling costs of bags and to improve the performance as much as possible. M3AL firstly adapts the multi-view self-representation learning to evacuate the shared and individual information of bags and to learn the shared/individual similarities between bags across/within views. Next, to avoid scrutinizing all the possible labels, M3AL introduces a new query strategy that leverages the shared and individual information, and the diverse instance distribution of bags across views, to select the most informative bag-label pair for query. Experimental studies on benchmark datasets show that M3AL can significantly reduce the query costs while achieve a better performance than other related competitive methods at the same cost.
\end{abstract}

Index Terms-Multi-view learning, multi-instance multi-label learning, active learning, commonality and individuality

\section{INTRODUCTION}

As a framework for modeling complex objects, Multiview Multi-instance Multi-label Learning (M3L) has attracted increasing interest in various applications [1], [2], [3], such as video annotation [4] and functional genomics [5], [6], [7]. In M3L, each object (or bag) contains one or more instances, is represented with different feature views, and is annotated with a set of non-exclusive semantic labels. For example, a video can be represented by the text view and image view, while each view contains multiple instances (i.e., persons and utterances), and is annotated with multiple labels, such as gate, grassland, football and so on. M3L aims to map multiple input feature views of bags onto the label space, and to identify the labels of a bag and/or its affiliated instances.

This work is supported by Natural Science Foundation of China (61872300, 62031003 and 62072380). Corresponding author: Jun Wang

G. Yu and J. Wang are with the School of Software and Joint SDU-NTU Centre for Artificial Intelligence Research (C-FAIR), Shandong University, China (e-mail: \{gxyu, kingjun\}@sdu.edu.cn)

Y. Xing is with the College of Computer and Information Sciences, Southwest University, China (e-mail: yyxing@swu.edu.cn)

C. Domeniconi is with the Department of Computer Science, George Mason University, USA (e-mail: carlotta@cs.gmu.edu)

$\mathrm{X}$. Zhang is with the King Abdullah University of Science and Technology, Thuwal, SA (e-mail: xiangliang.zhang@kaust.edu.sa)

Manuscript received May 14, 2020; Revised December 13, 2020; Accepted January 26, 2021.
Traditional M3L approaches generally require sufficient labeled M3 bags to train the model, given the multiplicity of M3 objects and the exponential power set of label groups for these objects. Unfortunately, collecting sufficient annotated bags is very expensive and even impractical. On the other hand, with the wide application of Internet of Things, it is easy to collect a large number of unlabeled M3 objects. However, existing M3L solutions [1], [8], [9], [2] cannot make full use of these unlabeled objects. One remedy is to incorporate active learning [10], [11], which tries to maximize the performance of a predictor by querying the most informative, and as few as possible, unlabeled objects or object-label pairs. Early studies of active learning mainly focus on the querying of objects with single view representation and concept labels, which can not be directly applied to the settings of multi-view, multiinstance, or multi-label data. In recent years, several active learning methods [12], [13], [14], [15] have been proposed to reduce the query costs of learning on single-view multi-instance multi-label (MIML) objects and on single-instance multi-view multi-label (MVML) objects. Some other approaches focus on querying the bag-label pairs to reduce the cost of scrutiny all possible labels of multi-label objects [16], [17]. However, in real-world applications, a complicated object can often be represented with different feature views and contains more than one instance. As a result, it is more difficult to explore active learning on M3 objects than on MIML and MVML objects.

Fig. 1 gives an example of how active learning can be used for the drug activity investigation and design. A drug (bag) is composed of many compounds (instances); when the drug enters into different organs (different feature views), its compounds can bind to different proteins and produce different re-activities, and that consequently results in different remedies and side effects. Considering the specific and common characteristics of these organs, the to-be-verified drug-reactivity pairs should not only focus on those shared by different organs, but also on pairs specific to individual organs. These pairs can be prioritized to biomedical experts for directional investigation and the obtained responses can improve the prediction as much as possible.

From this example, we summarize the challenges of active learning on M3 objects. Challenge 1: Each individual view may have its own most informative bags or bag-label pairs due to the heterogeneous feature views and distributions, and it is difficult to select the consensus most informative bag-label pairs across views for query. Challenge 2: Since single-view data may not credibly evaluate the informativeness of bags, how to leverage the shared and individual information of multiview data for credible evaluation is a non-trivial job. Challenge 


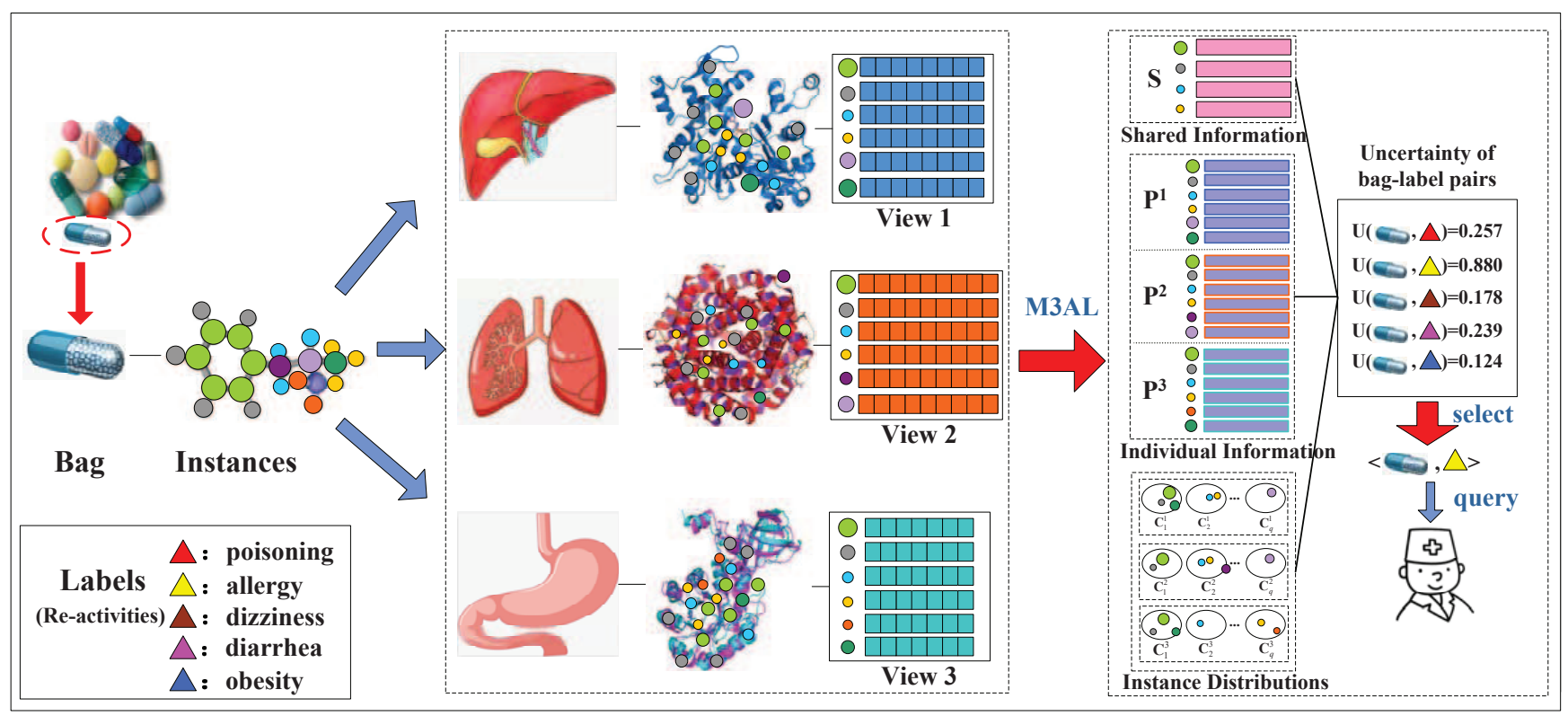

Fig. 1. An example of multi-view multi-instance multi-label active learning. The representation of drug compounds (instances) in each organ can be viewed as one view, and each drug is associated with multiple re-activities (i.e., labels). To mine the common and tissue-specific characteristics of liver, lung and stomach, M3AL explores the shared and individual information of drugs across these organs (views), and the diverse compound distributions of drugs across views to select the most informative drug-reactivity pair for query.

3: Unlike the single-instance active learning, how to leverage the associations between bags and instances, as well as the diverse instance distributions of bags across multiple views for informative query selection still lacks a principle way.

To address these challenges, we introduce a Multi-view Multi-instance Multi-label Active Learning (M3AL) approach. Fig. 11 illustrates the main idea of the proposed M3AL. M3AL firstly adapts the multi-view self-representation learning [18] to evacuate the shared and individual information of bags and to learn the global/individual similarity matrices between bags across/within views (for Challenge 2). To enhance the evacuated shared information, it additionally defines a multiview manifold regularization term based on the Hausdorff distance between bags and shared information matrix to explore the global similarity between bags. Next, M3AL introduces a new bag-label pair query strategy to avoid scrutinizing all the labels for a bag and to reduce the query cost. This strategy can leverage the shared and individual information of multiple data views to select the most informative and consensus bag-label pairs (for Challenge 1). Particularly, the consensus informative bag-label pair is jointly quantified based on the uncertainty of bag-label pairs using the evacuated common and individual information matrices, label correlations and the instance distribution across multiple views (for Challenge 1 and 3), respectively.

Our main contributions are summarized as follows:

1) We study a novel active learning setting, active learning on multi-view multi-instance multi-label objects, to reduce the labeling costs of the complex and challenging M3 objects, which has its distinctive challenges and applications. The proposed M3AL can work on M3 objects without instance-level labels.

2) M3AL introduces a new bag-label pair selection criterion that evacuates and leverages the individual and shared information of bags across multiple views, label correlations and diverse instance distributions of the respective bags in different views, to select the informative bag-label pairs for query.

3) Experimental study shows that our M3AL outperforms existing active learning solutions [13], [12], [14], [15], [19], [20] on benchmark datasets, and it also confirms the advantage of the proposed bag-label pair selection criterion to existing criteria proposed for single-view MIML objects and single-instance MVML objects.

The rest of this paper is organized as follows: Section III reviews the related work of active learning for MIML and MVML objects; Section III elaborates on the proposed M3AL. The experimental results and analysis are reported in Section IV] and Section $\mathrm{V}$ concludes the work and future pursues.

\section{RELATED WORKS}

Active learning [21], as an important branch of machine learning, aims to query unlabeled objects as few as possible while improve the performance as much as possible. Under this promise, the key task is to design the query strategy to select the most informative object or object-label pair for query. Diverse criteria have been introduced for the query selection, such as diversity, density, uncertainty, and so on. Traditional active learning strategies focus on objects represented with single feature view, annotated with single labels [22]. The query of object-label pair proves to be an effective and efficient strategy in multi-label active learning, since the scrutiny of all possible labels of a multi-label object is too time consuming and the labels are inter-dependent [16], [17]. Given that, M3AL selects the most informative bag-label pairs for query.

Compared with the heavy study of active learning on singleinstance multi-label (or multi-class) objects [23], [17], [24], [25], active learning on single-view MIML objects and singleinstance MVML objects is less explored, and not to mention 
active learning on M3 objects. That is due to a non-exclusive subset of labels should be reliably queried (multi-label), the unknown instance-level labels and the variety associations between bags and instances (multi-instance), or the complicated inter-relationships between bags across views (multi-view) In the following two subsections, we give a brief review of approaches for active learning in conjunction with MIML or MVML objects, and then we elaborate on how active learning on $\mathrm{M} 3$ objects differs.

\section{A. Active Learning with MIML}

Given the widely witnessed MIML objects, several approaches [14], [15], [12], [13] have been proposed to reduce the query costs of active learning on MIML objects at the bag level or the more granular instance level. Retz et al. [13] combined MIMLSVM [26] with the $k$-Medoids clustering algorithm by transforming multiple instances into a single-instance representation, and then they selected the most informative bags for query. To reduce the query cost of MIML, Huang et al. [12] proposed an active learning approach called MIML$\mathrm{AL}$, which targets to select the most valuable bag-label pairs by exploiting diversity and uncertainty in both the input and output spaces, and acquires more precise instance-label relation from the oracle without additional cost. Inspired by the observation that one can annotate a bag-label pair via annotating the responsible instances of the target bag, Chen et al. [14] presented a novel two-stage cost-effective multilabel active learning framework called CMAL. CMAL firstly selects the most uncertain bag-label pair by leveraging label correlation and label space sparsity of bags; then it queries the most plausible positive instance-label pair (estimated by an instance-level classifier) of the selected bag-label pair to reduce the labeling costs. Yu et al. [27] extended CMAL by further incorporating the representativeness of bags to select more informative bag-label pairs. Carboneau et al. [15] introduced two strategies (Aggregated Information, AGIN; ClusteringBased Aggregative Sampling, C-BAS) for multiple instance active learning in instance classification problems. AGIN selects the most informative bags for query, whose informativeness is aggregated from its classifier uncertain instances. C-BAS applies the divisive hierarchical clustering to group instances, and then it queries the bag by considering bag labels and the proportion of labels that remains to be discovered in clusters.

These active learning solutions all aim to reduce the query costs in a single-view setting. Some of them [13], [15] have to scrutinize the selected bag with respect to all the labels, which result in unnecessary cost, given the label sparsity and correlations of multi-label data. In addition, for many real-world applications, MIML objects are often encoded with different feature views. Since individual views may have different distributions, each view has its own most informative bags or bag-label pairs. So how to judge the most informative bags across views is still unclear.

\section{B. Active Learning with $M V M L$}

Several multi-view multi-label active learning approaches have been suggested to select the most uncertain objects and labels across multiple views for query. Wang and Zhou [28] firstly characterized the object complexity of multi-view active learning; they integrated multi-view active learning with semi-supervised learning to reduce the object complexity. Other multi-view active learning approaches [19], [13] aim to reduce the query costs of multi-view objects by integrating the uncertainty of objects, and labels shared by multiple views and specific to each view, through different fusion strategies. Zhang et al. [20] used the expected entropy and a new variance-based strategy to measure the generalization error of each samplelabel pair rated by multiple views and by each specific view, and then combined those two measured errors to select the informative sample-label pairs for query.

Despite these progresses, the aforementioned active learning with MVML solutions still can not account for objects with various instances, which provide additional hints on how to select the bag for query and reduce the query cost on complex bags. Furthermore, these active learning solutions still do not make good use of shared and individual information of multiple data views, which are essential for effective multiview data mining [29], [30], [31], [32], [33]. In this paper, we study active learning in a more general setting, where each object is represented with different feature views and includes diverse instances per view. Our proposed M3AL can not only mine the shared and individual information of multiview data to select the most informative bag-label pairs, but also consider the diverse instance distributions of bags across multiple views. M3AL achieves a better performance with a lower query cost than the state-of-the-art solutions on public MVML/MIML/M3L datasets.

\section{THE PRoposed M3AL}

\section{A. Problem Formulation}

Suppose $n$ bags are represented by $V$ feature views, and $\mathcal{D}_{l}^{v}=\left\{\left(\mathcal{X}_{1}^{v}, \mathbf{Y}_{1}\right),\left(\mathcal{X}_{2}^{v}, \mathbf{Y}_{2}\right), \cdots,\left(\mathcal{X}_{l}^{v}, \mathbf{Y}_{l}\right)\right\}$ represents $l$ labeled bags in the $v$-th view. The $i$-th bag in the $v$-th view has $m_{i}^{v} \geq 1$ instances, i.e., $\mathcal{X}_{i}^{v}=\left\{\mathbf{x}_{i, 1}^{v}, \mathbf{x}_{i, 2}^{v}, \cdots, \mathbf{x}_{i, m_{i}^{v}}^{v}\right\}(1 \leq$ $v \leq V)$ where $\mathbf{x}^{v} \in \mathbb{R}^{d_{v}}(v=1,2, \cdots, V)$ is the feature space of instances in the $v$-th view. $\mathbf{Y}_{i} \in \mathbb{R}^{q}$ and $\mathbf{y}_{k} \in \mathbb{R}^{q}$ store the known labels of $\mathcal{X}_{i}^{v}$ and instances $\mathbf{x}_{k}$, respectively. $\mathbf{Y}_{i c}=1$ if $\mathcal{X}_{i}^{v}$ is annotated with the $c$-th label; $\mathbf{Y}_{i c}=-1$ otherwise. $\mathbf{y}_{k}$ follows the same protocol to encode the labels of instances. All bags (instances) belong to the same label space. Suppose $\mathcal{D}_{u}^{v}=\left\{\mathcal{X}_{l+1}^{v}, \cdots, \mathcal{X}_{l+u}^{v}\right\}$ collects unlabeled bags of the $v$-th view. M3AL aims to select the most informative bag-label pairs by simultaneously considering the shared and individual information across multiple views, and the diverse instance distributions of bags in different views for query, whose responses (positive or negative pairs) can improve the predictor as much as possible. In the following subsections, we elaborate on how M3AL makes it.

\section{B. Extracting the Shared and Individual Information}

Single-view multi-instance active learning mainly focuses on the intra-relation between bags within the same view. In contrast, multi-view multi-instance active learning should account for the inter-relation between bags across views. On 
the one hand, due to the heterogeneous feature views, there may be inconsistencies between the selected informative bag-label pairs across views. On the other hand, since each view partially represents objects from different perspectives, the selected bag-label pairs derived from a single view may be not so reliable. In other words, both the individuality and commonality information of multi-view data should be accounted for active learning on multi-view data. To make this, M3AL firstly extends the multi-view self-representation learning [34], [18] to extract the individuality and commonality of multi-view bags as follows:

$$
\begin{aligned}
\Theta\left(\left\{\mathbf{P}^{v}\right\}_{v=1}^{V}, \mathbf{S}\right)=\min & \frac{1}{V} \sum_{v=1}^{V}\left\|\mathbf{X}^{v}-\left(\mathbf{S}+\mathbf{P}^{v}\right) \mathbf{X}^{v}\right\|_{F}^{2} \\
& +\lambda_{1} \Phi_{1}\left(\left\{\mathbf{P}^{v}\right\}_{v=1}^{V}\right)+\lambda_{2} \Phi_{2}(\mathbf{S})
\end{aligned}
$$

where $\mathbf{X}^{v} \in \mathbb{R}^{n \times d_{v}}$ is the feature data matrix of $n$ bags in the $v$-th view. Since an M3 object can include more than one instance, we firstly use the canonical approach adopted in [35] to transform multiple instance feature vectors of a bag $\left(\mathcal{X}_{i}^{v}\right)$ into a single feature vector of this bag $\left(\mathbf{X}_{i}^{v}\right)$. Particularly, the instances of bags are first divided into groups by clustering, and then the constructive clustering based ensemble is utilized to represent the bag as a single feature vector based on the clustering results. This representation can give the same feature size of bags across views. The first term of Eq. (1) is to capture the shared and individual feature information of bags across views. The second term is to enforce the difference among individuality encoding matrices $\left(\left\{\mathbf{P}^{v}\right\}_{v=1}^{V} \in \mathbb{R}^{n \times n}\right)$, and the third term is to pursue the commonality encoding matrix $\left(\mathbf{S} \in \mathbb{R}^{n \times n}\right)$ across views.

To extract the individuality of each view, we choose the widely used Hilbert-Schmidt Independence Criterion (HSIC) [36] to quantify the linear and nonlinear inter-dependency between $\mathbf{P}^{v}$ and $\mathbf{P}^{v^{\prime}}\left(v \neq v^{\prime}\right)$. HSIC computes the squared norm of the cross-covariance operator over $\mathbf{P}^{v}$ and $\mathbf{P}^{v^{\prime}}$ in the Hilbert Kernel space to estimate the dependency. The empirical HSIC does not have to explicitly compute the joint distribution of $\mathbf{P}^{v}$ and $\mathbf{P}^{v^{\prime}}$, it is given by

$$
\operatorname{HSIC}\left(\mathbf{P}^{v}, \mathbf{P}^{v^{\prime}}\right)=(n-1)^{-2} \operatorname{tr}\left(\mathbf{K}^{v} \mathbf{L} \mathbf{K}^{v^{\prime}} \mathbf{L}\right)
$$

where $\mathbf{K}^{v} \in \mathbb{R}^{n \times n}$ and $\mathbf{K}^{v^{\prime}} \in \mathbb{R}^{n \times n}$ are used to measure the kernel induced similarity between vectors of $\mathbf{P}^{v}$ and of $\mathbf{P}^{v^{\prime}}$, respectively. In this paper, we use the inner product kernel to specify $\mathbf{K}^{v}=\left(\mathbf{P}^{v}\right)^{T} \mathbf{P}^{v}(\forall v \in\{1,2, \cdots, V\}) . \mathbf{L}(i, j)=$ $\delta_{i j}-1 / n$ is the centering matrix in the kernel space, $\delta_{i j}=1$ if $i=j ; \delta_{i j}=0$ otherwise. To reduce the redundancy between different $\left\{\mathbf{P}^{v}\right\}_{v=1}^{V}$, we minimize the overall HSIC on $V$ views as:

$$
\begin{array}{r}
\Phi_{1}\left(\left\{\mathbf{P}^{v}\right\}_{v=1}^{V}\right)=\sum_{v=1, v \neq v^{\prime}}^{V} \operatorname{HSIC}\left(\mathbf{P}^{v}, \mathbf{P}^{v^{\prime}}\right) \\
=\sum_{v=1}^{V} \operatorname{tr}\left(\mathbf{P}^{v} \widetilde{\mathbf{K}}^{v}\left(\mathbf{P}^{v}\right)^{T}\right) \\
\text { s.t. } \quad \widetilde{\mathbf{K}}^{v}=(n-1)^{-2} \sum_{v^{\prime}=1, v^{\prime} \neq v}^{V} \mathbf{L K}^{v^{\prime} \mathbf{L}}
\end{array}
$$

Both the inter(intra)-relation between bags and those between instances of a bag can be used to improve the performance of M3L [37], [3]. To mine more shared information, the third term in Eq. 11 enforces the consistency among different views. For this purpose, we first quantify the similarity between bags within each view based on the widely used average Hausdorff distance [13], [37] as follows:

$$
\begin{array}{r}
\mathbf{H}^{v}(i, j)=\frac{\sum_{\mathbf{x}_{a}^{v} \in \mathcal{X}_{i}^{v}} \min _{\mathbf{x}_{b}^{v} \in \mathcal{X}_{j}^{v}} d\left(\mathbf{x}_{a}^{v}, \mathbf{x}_{b}^{v}\right)}{\left|\mathcal{X}_{i}^{v}\right|+\left|\mathcal{X}_{j}^{v}\right|} \\
+\frac{\sum_{\mathbf{x}_{b}^{v} \in \mathcal{X}_{j}^{v}} \min _{\mathbf{x}_{a}^{v} \in \mathcal{X}_{i}^{v}} d\left(\mathbf{x}_{a}^{v}, \mathbf{x}_{b}^{v}\right)}{\left|\mathcal{X}_{i}^{v}\right|+\left|\mathcal{X}_{j}^{v}\right|}
\end{array}
$$

where $d\left(\mathbf{x}_{a}^{v}, \mathbf{x}_{b}^{v}\right)$ represents the Euclidean distance between two instances $\left(\mathbf{x}_{a}^{v}\right.$ and $\mathbf{x}_{b}^{v}$ ) of two bags. The average Hausdorff distance takes into accounts more geometric relations between instances of two bags than the minimum and maximum Hausdorff distances [38], [37]. Other distance metrics can also be used here. Unlike the Euclidean distance for singleinstance representation, the Hausdorff distance is canonically used for multi-instance learning, it considers the instance composition for respective bags. Hausdorff distance can also reduce the information loss caused by the representation transformation of original bags. In fact, Retz et al. [13] had also shown the advantage of Hausdorff distance for measuring the similarity between bags. Then, we define the Gaussian heat kernel $\mathbf{R}^{v}(i, j)=\exp \left(-\mathbf{H}^{v}(i, j) / \sigma_{v}^{2}\right)$ to quantify the similarity between bags, and $\sigma_{v}$ is set to the average of $\mathbf{H}^{v}$. Next, we build a manifold regularization-based term [39] over all views to extract the commonality information matrix as follows:

$$
\begin{array}{r}
\Phi_{2}(\mathbf{S})=\sum_{v=1}^{V}\|\mathbf{S}(i, \cdot)-\mathbf{S}(j, \cdot)\| \mathbf{R}^{v}(i, j) \\
=\operatorname{tr}\left(\mathbf{S}\left(\frac{1}{V} \sum_{v=1}^{V}\left(\mathbf{D}^{v}-\mathbf{R}^{v}\right) \mathbf{S}^{T}\right)\right.
\end{array}
$$

where $\mathbf{D}^{v}$ is a diagonal matrix with diagonal entries equal to respective row sum of $\mathbf{R}^{v}$. Since $\mathbf{S}$ is shared across $V$ views and regularized by the geometric distributions of bags and instances therein per view, minimizing $\Phi_{2}(\mathbf{S})$ can strengthen the shared information evacuation from multiple views.

To this end, Eq. (1) can be reformulated as follows:

$$
\begin{array}{r}
\Theta\left(\left\{\mathbf{P}^{v}\right\}_{v=1}^{V}, \mathbf{S}\right)=\min \frac{1}{V} \sum_{v=1}^{V}\left\|\mathbf{X}^{v}-\left(\mathbf{S}+\mathbf{P}^{v}\right) \mathbf{X}^{v}\right\|_{F}^{2} \\
+\lambda_{1} \sum_{v=1}^{V} \operatorname{tr}\left(\mathbf{P}^{v} \widetilde{\mathbf{K}}^{v}\left(\mathbf{P}^{v}\right)^{T}\right) \\
+\frac{\lambda_{2}}{V} \operatorname{tr}\left(\mathbf{S} \sum_{v=1}^{V}\left(\mathbf{D}^{v}-\mathbf{R}^{v}\right) \mathbf{S}^{T}\right.
\end{array}
$$

$\mathbf{S}$ and $\left\{\mathbf{P}^{v}\right\}_{v=1}^{V}$ can be viewed as the global similarity between bags across multiple data views, and as the individual similarity matrices between bags within each view, respectively. Unlike the previous multi-view self-representation learning that either focus on commonality [34], or more on individuality without a concrete use of data manifolds from multiple views [18], our multi-view self-representation learning keeps a balance of them. 
The detailed optimization of $\mathbf{S}$ and $\mathbf{P}^{v}$, and the convergence proof this optimization are given in the Supplementary file. Both $\mathbf{S}$ and $\mathbf{P}^{v}$ will be used to select the most informative bag-label pairs for query in the next subsection.

\section{Selecting Informative Bag-Label Pair across Views}

Unlike single-label (or multi-class) active learning, multilabel active learning requires the oracle to scrutinize all the possible label subsets, and then to annotate all the relevant labels to the object. This exhaustive scrutiny is too expensive and ultimately impossible. It is recognized that query bag-label pair, instead of labels or bags only, can reduce the query cost and also boost the performance [16], [12], [14]. To select the most informative bag-label pairs across multiple views in M3L scenario, we advocate to quantify the unified informativeness of a bag-label pair $\langle i, c\rangle$ by leveraging the shared and individual information, and the instance distributions of the $i$-th bag across views as follows:

$$
\begin{aligned}
\mathbf{Q}(i, c)= & D B(i) *\left(U_{b}(\widehat{\mathbf{S}}, i, c)+\alpha \frac{1}{V} \sum_{v=1}^{V} U_{b}\left(\widehat{\mathbf{P}}^{v}, i, c\right)\right), \\
& (1 \leq i \leq u, 1 \leq c \leq q)
\end{aligned}
$$

where $U_{b}(\widehat{\mathbf{S}}, i, c)$ represents the shared informativeness of the bag-label pair $\langle i, c\rangle$ from multiple views, and $U_{b}\left(\widehat{\mathbf{P}}^{v}, i, c\right)$ represents the individual informativeness of this pair from the $v$-th view. $\alpha \in[0,1]$ is a parameter to balance the importance of these two types of informativeness. $D B(i)$ is used to quantify the diversity of the $i$-th bag by capturing its instance distributions in different views. Previous multi-view self-representation learning based solutions directly use $\mathbf{S}$ and $\left\{\mathbf{P}^{v}\right\}_{v=1}^{V}$ for follow-up learning tasks [29], [18], [32]. Here, to reduce the data fidelity when selecting bag-label pairs, we take $\widehat{\mathbf{S}}=\frac{1}{V} \sum_{v=1}^{V} \mathbf{X}^{v} \mathbf{S}$ and $\widehat{\mathbf{P}}^{v}=\mathbf{X}^{v} \mathbf{P}^{v}$ as the input features to leverage the commonality and individuality of bags across views for selecting bag-label pairs.

We want to remark that although the first two informativeness of the bag-label pair have the same formulation, they account for different aspects (commonality and individuality) of M3 objects. The same bag in different views may have different numbers of instances, so the aggregated uncertainties from instances to their originating bag can only be quantified per view. Different from existing active learning with MIML and with MVML, Eq. (7) simultaneously accounts for the informativeness from the instance-space, bag-space and multiple views, whereas the former two types of active learning only consider one or two of them. Therefore, M3AL handles a more complicated active learning problem.

$\mathbf{Q} \in \mathbb{R}^{u \times q}$ stores the unified informativeness of $u$ unlabeled bags for $q$ labels. We can select the most informative bag-label pair for query as follows:

$$
<\mathcal{X}_{i^{*}}, c^{*}>=\max _{i \in\{1, \cdots, u\}} \max _{c \in\{1, \cdots, q\}} \mathbf{Q}(i, c)
$$

1) Uncertainty of Bag-Label Pair across Views: We adopt the widely used uncertainty criterion to measure the informativeness of a bag-label pair, and to select the bag-label pair with a high uncertainty. The more uncertain of a bag-label pair, the more the pair (when queried) can reduce the model ambiguity. We first use the labeled bags to train a bag-level classifier, here the SVM classifier is used as an example. For SVM, the uncertainty of a bag-label pair can be measured by the distance between the bag and the decision boundary. Suppose the decision boundary w.r.t. the $c$-th label is defined as $F_{c}(\mathbf{X})=0$, and the distance between the $i$-th bag and this label is defined as $\left|F_{c}\left(\mathbf{X}_{i}\right)\right|$. Next, we can convert this distance to measure the uncertainty of a bag-label pair as follows:

$$
U_{b}(\widehat{\mathbf{S}}, i, c)=\frac{1}{\left|F_{c}\left(\widehat{\mathbf{S}}_{i}\right)\right|} \times\left(1-\frac{\sum_{k=1}^{q} \mathbf{Y}^{+}(i, k) \mathbf{W}(k, c)}{\sum_{k=1}^{q} \mathbf{Y}^{+}(i, k)}\right)
$$

The larger the value of $\frac{1}{\left|F_{c}\left(\widehat{\mathbf{S}}_{i}\right)\right|}$ is, the more uncertain the bag-label pair is. This uncertainty measurement is widely used as a basis to select the most uncertain objects or bag-label pairs [15], [14]. The second term in Eq. (7) is introduced to make use of the label correlations, which can also reduce the query cost. For example, if $c$ has large correlations with other queried labels of the $i$-th bag, then we should give low priority to $\langle i, c\rangle$, since the uncertainty of $\langle i, c\rangle$ can be reduced by these queried labels. $\mathbf{Y}^{+} \in \mathbb{R}^{n \times q}$ only encodes the positive labels of bags. We adopt the widely used linear kernel to convert $\widehat{\mathbf{S}}\left(\widehat{\mathbf{P}}^{v}\right)$ into a kernel matrix for SVM.

Since the labeled bags are too limited to reliably estimate $\mathbf{W}(k, c)$, we re-estimate $\mathbf{W}(k, c)$ after each query to alleviate this issue as follows:

$$
\mathbf{W}(k, c)=\frac{\sum_{i=1}^{l+u} I\left[\mathbf{Y}_{i, c}=+1, \mathbf{Y}_{i, k}=+1\right]}{\sum_{i=1}^{l+u} I\left[\mathbf{Y}_{i, k}=+1\right]}
$$

where $I[\cdot]$ is an indicator function, $I[\cdot]=1$ if the condition is met; $I[\cdot]=0$ otherwise. We want to admit that other estimations of label correlations or dependencies [40], [12] can also be adopted and may further boost the bag-label pair selection, whose investigation is not the focus of this paper. Following the same procedure as Eq. (7), we can also compute the uncertainty of $U\left(\widehat{\mathbf{P}}^{v}, i, c\right)$ from each individuality matrix.

2) The Diversity of Bags from Instances across Views: In M3L setting, the instance distributions within a bag across multiple views can provide supplementary information for bag selection [15]. In other words, if all the instances of a bag have the same distribution or labels, such bag has a lower diversity and is less expected to be selected for query. On the other hand, querying a bag with diverse instance distributions can greatly reduce the uncertainty of the model, and thus may boost the predictor. We adopt the widely used $k$-means clustering to explore the instance diversity of a bag across multiple views and to measure the instance distributions within a bag across multiple views. Particularly, we group all unlabeled instances in each view into $C$ clusters, and measure the instance distribution of a bag in the $v$-th view according to the instance clustering results as follows:

$$
D B^{v}(i)=\frac{1}{C} \sum_{c=1}^{C} \frac{\beta_{c}^{v}(i) \log \left(\beta_{c}^{v}(i)\right)+\left(1-\beta_{c}^{v}(i)\right) \log \left(1-\beta_{c}^{v}(i)\right)}{\log (0.5)}
$$

where $\beta_{c}^{v}(i)$ counts the proportion of instances in the $i$-th unlabeled bag distributed in the $c$-th cluster of the $v$-th view, it can be calculated by the ratio between the number of instances 
distributed in the $c$-th cluster and the number of instances of the $i$-th bag in the $v$-th view.

The same bag in multiple views differs in affiliated instances and instance distributions; thus, its instance cluster representations are also distinct across views. $D B^{v}(i)$ reflects the diversity of the $i$-th bag in the $v$-th view. A larger $D B^{v}(i)$ means the more diverse distributions of multiple instances of a bag. We can average the diversity of the $i$-th bag across multiple views and acquire the overall bag diversity as follows:

$$
D B(i)=\frac{1}{V} \sum_{v=1}^{V} D B^{v}(i)
$$

where $D B(i)$ aggregates instance distributions of the $i$-th bag across multiple views, and a larger value of $D B(i)$ shows a larger diversity of the $i$-th bag, which can coordinate the selection of informative bags. Our following experiments will confirm the benefit of mining instance distributions within a bag across multiple views.

To this end, by Eq. (7), M3AL can select the most informative bag-label pairs across multiple views by simultaneously exploiting the shared and individual information of bags, and the diverse instance distributions within bags across views.

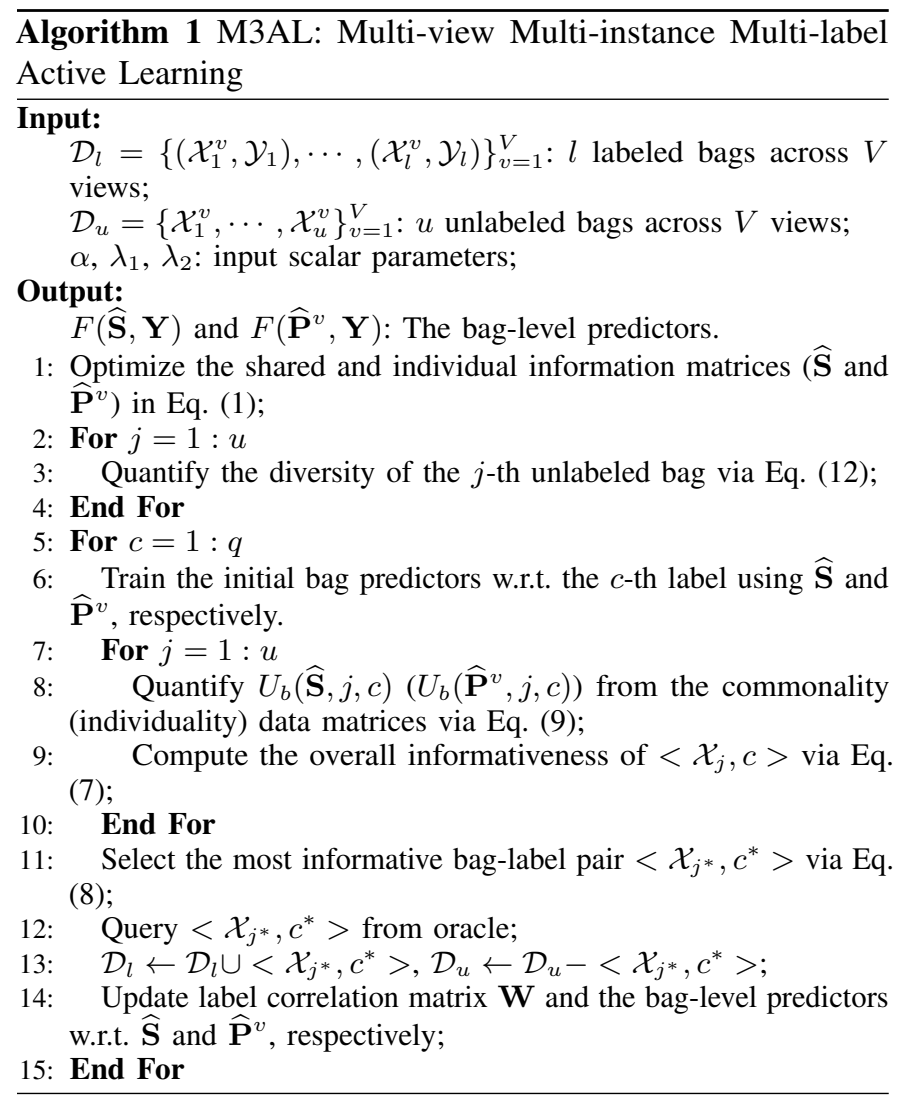

Algorithm 1 summarizes the pseudo-code of M3AL. M3AL first learns the shared and individual information of bags across multiple views (i.e., $\mathbf{S}$ and $\left\{\mathbf{P}^{v}\right\}_{v=1}^{V}$ ) based on multiview self-representation learning (step 1), respectively. It then quantifies the instance distributions of each unlabeled bag across multiple views to measure the bag diversity via Eq. (12) (step 3). After that, M3AL trains the initial bag predictors using the shared and individual information of $l$ labeled bags w.r.t each label, respectively (step 6). Next, it calculates the overall uncertainty by combing the uncertainty of shared and individual information of the bag, and its diverse instance distributions (step 8-9). M3AL finally uses the combined uncertainty to select the most informative bag-label pair for query (step 1112), updates label correlation matrix and the bag-level predictors with obtained query responses (step 13-14).

\section{Time Complexity Analysis}

In this subsection, we analyze the time complexity of the proposed M3AL. Suppose there are $V$ views, $n$ bags, $m$ instances and $d_{v}$ features for $\mathbf{X}^{v}$, the time complexity for computing the average Hausdorff distance, and learning $\mathbf{S}$ and $\left\{\mathbf{P}^{v}\right\}_{v=1}^{V}$ in each iteration are $O\left(V n^{2} m d_{v}\right), O\left(V n^{2} d_{v}\right)$ and $O\left(V n^{2} d_{v}\right)$, respectively. Let the number of required iterations for optimization be $t_{1}$, the overall time complexity for solving $\mathbf{S}$ and $\left\{\mathbf{P}^{v}\right\}_{v=1}^{V}$ optimization is $O\left(2 t_{1} V n^{2} d_{v}+V n^{2} m d_{v}\right)$. In practice, our empirical study shows that the optimization converges within 40 iterations on our used datasets. Suppose $t_{2}$ is the number of queries, the time complexity of our adopted SVM [41] in the $v$-th view is $O\left(q n^{2} d_{v}\right) \sim O\left(q n^{3} d_{v}\right)$ Besides, M3AL utilizes $k$-means clustering to explore the instance diversity of a bag across multiple views. The time complexity of this process is $O\left(t_{3} V C m d_{v}\right)$, where $C$ and $t_{3}$ are the number of clusters and iterations, respectively. Thus the time complexity of M3AL for bag-label pairs selection is $O\left(t_{2} V q n^{2} d_{v}+t_{3} V C m d_{v}\right) \sim O\left(t_{2} V q n^{3} d_{v}+t_{3} V C m d_{v}\right)$. To sum up, the overall time complexity of our proposed model is $O\left(\left(2 t_{1} n^{2}+n^{2} m+t_{2} q n^{2}+t_{3} C m\right) V d_{v}\right) \sim O\left(\left(2 t_{1} n^{2}+\right.\right.$ $\left.\left.n^{2} m+t_{2} q n^{3}+t_{3} C m\right) V d_{v}\right)$. More efficient matrix factorization techniques [42] can be adopted here to speedup M3AL on large multi-view datasets. Due to the sparsity of multi-view data, the above matrix computations can be efficiently made in practice.

\section{EXPERIMENTS}

\section{A. Experimental Setup}

1) Datasets: We use diverse benchmark datasets, including four single-view multi-instance multi-label datasets with instance labels [43], [44], three single-instance multi-view multi-label datasets [45], [46] and one real-world M3 data [6], to evaluate the performance of our proposed M3AL. These datasets are from different domains and with different scales, and most of them are also used by compared methods. Table I lists the details of these datasets. In this paper, we adopted a unified view partition and bag transformation technique [37], [47] to test all multi-view active learning methods (i.e., M3AL, M2DAL [19] and MultiAL [20]). For the four single-view datasets, we divide the original features of each bag into two views by randomly selecting half features for one view and the remaining features for the other view, to test multi-view based solutions. We directly adopt the three multi-view datasets and the multi-view multi-instance Isoform dataset for multi-view active learning algorithms, while we concatenate feature views of multi-view dataset into a single one for single-view active learning methods. 
TABLE I

STATISTICS OF EIGHT DATASETS USED FOR THE EXPERIMENTS. AVGBI IS THE AVERAGE NUMBER OF INSTANCES PER BAG, AVGBL IS THE AVERAGE NUMBER OF LABELS PER BAG, AND $V$ IS THE NUMBER OF VIEWS.

\begin{tabular}{l|rrrrrr}
\hline Dataset & bag & instance & label & avgBI & avgBL & $V$ \\
\hline Letter Frost & 144 & 565 & 26 & 3.9 & 3.6 & 1 \\
Letter Carroll & 166 & 717 & 26 & 4.3 & 3.9 & 1 \\
MSRC v2 & 591 & 1758 & 23 & 3.0 & 2.5 & 1 \\
Birds & 548 & 10232 & 13 & 18.7 & 2.1 & 1 \\
\hline Emotions & 593 & 593 & 6 & 1.0 & 1.9 & 2 \\
Yeast & 2417 & 2417 & 14 & 1.0 & 4.2 & 2 \\
Pascal & 9963 & 9963 & 20 & 1.0 & 3.4 & 3 \\
\hline Isoform & 2000 & 7907 & 50 & 4.0 & 3.9 & 2 \\
\hline
\end{tabular}

2) Compared Methods: To investigate the performance of the proposed M3AL, three experiments are designed. In the first experiment, we compare M3AL against four representative and related single-view MIML methods and two single-instance MVML approaches to investigate the performance of M3AL on single-view and multi-view settings, respectively. The second experiment investigates the contribution of different components of M3AL for measuring the uncertainty of baglabel pairs. The third experiment studies the sensitivity of input parameters of M3AL.

In the first experiment, we compare M3AL against MIML and MVML approaches with different active learning strategies as follows:

(i) MidSelect [13] is a single-view MIML method, it uses MIMLSVM as the classifier by transforming the multiple instances into a single one to select the most uncertain baglabel pairs.

(ii) MIML-AL [12] targets to select the most valuable baglabel pairs by exploiting diversity and uncertainty in both the input and output spaces, and acquires more precise instancelabel relation from the oracle without extra cost.

(iii) CMAL [14] is another single-view MIML active learning method, it selects the most uncertain example-label pair by querying its most plausible positive instance-label pairs.

(iv) AGIN [15] is a single-view MIML solution also, it aims at querying the bags with the highest informativeness score by aggregating the informativeness scores of instances.

(v) Multi-view 2DAL (M2DAL) [19] is an MVML-based active learning solution, it selects the most informative samplelabel pairs by combing the overall sample predictions across multiple views based on the super-kernel fusion, and the intraview bag predictions based on min-max strategy.

(vi) MultiAL [20] is another MVML-based solution, it performs multi-view active learning by unifying view-specific sample uncertainty based on information entropy, and variance-based shared uncertainty across multiple views.

The other input parameters of these compared methods are configured as the authors suggested in the original papers or shared codes, which are specified in Table S1 of the Supplementary file. For a fair comparison, we use $q$ one-vs-rest SVM (implemented with LibSVM [41]) as the base classifier for the evaluation of all compared methods, and adopt the default input values of LibSVM for SVM.

To evaluate the effectiveness of M3AL, we adopt the representative and widely used multi-label evaluation metric Average Precision [12], [14], which aims to evaluate the average fraction of labels ranked above a particular label. The formal definition of Average Precision is as follows:

$$
A P(f)=\frac{1}{n} \sum_{i=1}^{n} \frac{1}{\left|\mathcal{Y}_{i}\right|} \sum_{c_{1} \in \mathcal{Y}_{i}} \frac{\left|\left\{c_{2} \in \mathcal{Y}_{i} \mid \operatorname{rank}\left(F\left(i, c_{2}\right)\right) \leq \operatorname{rank}\left(f\left(i, c_{1}\right)\right)\right\}\right|}{\operatorname{rank}\left(F\left(i, c_{1}\right)\right)}
$$

where $n$ is the total number of bags, $\mathcal{Y}_{i}$ is the label set of the $i$-th bag. $\operatorname{rank}(F(i, \cdot))$ represents the ranking function, which sorts all the predicted labels of a bag in descending order by $F(i, \cdot) \in \mathbb{R}^{q}$. The larger the value of Average Precision, the better the performance is.

\section{B. Results Comparison with Other Methods}

To explore the performance of M3AL, we randomly partition samples of each dataset into a training set $(70 \%)$ and a testing set $(30 \%)$. For the training set, we again randomly select $5 \%$ samples as the initial labeled data $\left(\mathcal{D}_{l}^{v}\right)$ and the remaining as unlabeled data $\left(\mathcal{D}_{u}^{v}\right)$ for query. The above partitions are independently repeated 10 times and Fig. 2 shows the average results of compared methods on different datasets.

From results in these figures, we have the following observations:

(i) Multi-view vs. Single-view: M3AL outperforms singleview MIML active learning solutions (AGIN, MIML-AL, MidSelect and CMAL) across different types of datasets. This fact suggests that M3AL can leverage the commonality and individuality information of bags across views to select baglabel pairs. In contrast, AGIN, MIML-AL, MidSelect and CMAL can only use the complementary information of bags by concatenating features. In addition, they mine the instance information by directly using the instance-level predictions, while M3AL aggregates various instance distributions of each bag across multiple views to guide the selection of bag-label pair for query. The performance margin between them indicates the effectiveness of M3AL on exploring and employing instance information for active learning on multi-view and multi-instance data. For these two advantages, although M3AL and CMAL adopt a similar bag-label uncertainty criterion, and both exploit label correlations to query the bag-label pairs, M3AL still significantly outperforms CMAL. In other words, CMAL mainly explores the commonality information of multi-view data by concatenating them and lacks a concrete use of instance distributions. M3AL also gives a much better performance than compared methods on the real M3 Isoform dataset. This fact again confirms M3AL can effectively reduce the labeling costs of complex M3 objects. The results of MIML-AL show some fluctuation as the number of queries increase on several datasets, and the possible reasons for such pattern is mainly caused by two factors. MIML-AL uses the rank loss for the bag selection, while the labels of these datasets are relatively sparse which may cause misrepresentation of unexpected label information; MIML-AL queries bag-label pairs based on the rank loss of key instances of a bag, and the selection of key instances also impacts the model performance in multi-instance setting, especially for datasets with more average instances per bag. We observe that MIML-AL manifests a reduced performance on the real M3 dataset. The possible reason is that the data features of Isoform objects are very sparse, which skew the ranking-loss 


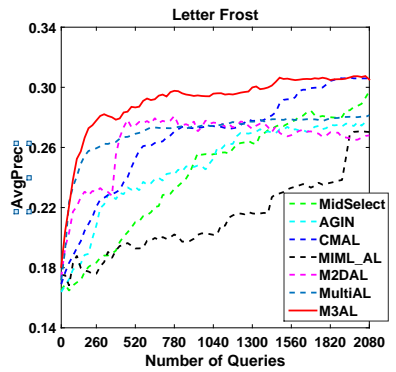

(a) Letter Frost

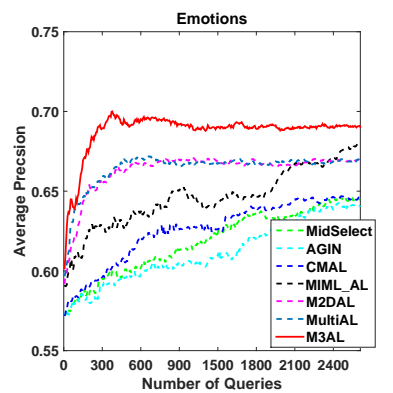

(e) Emotions

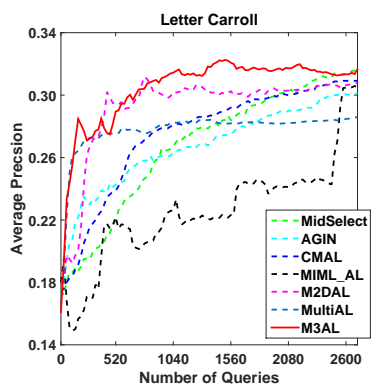

(b) Letter Carroll

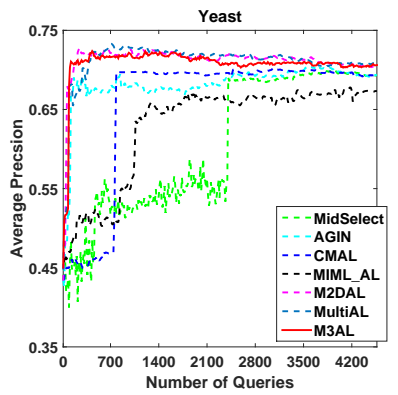

(f) Yeast

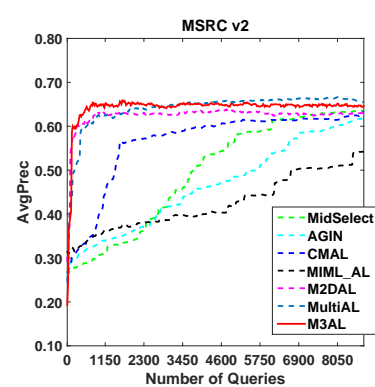

(c) MSRC v2

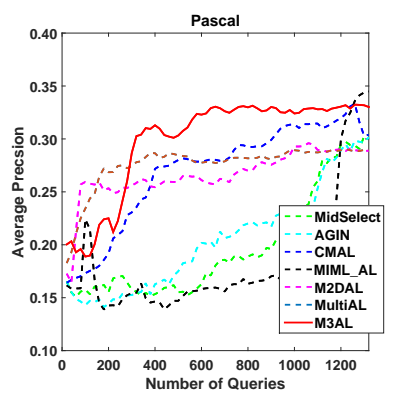

(g) Pascal

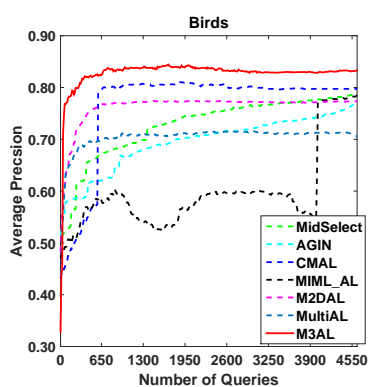

(d) Birds

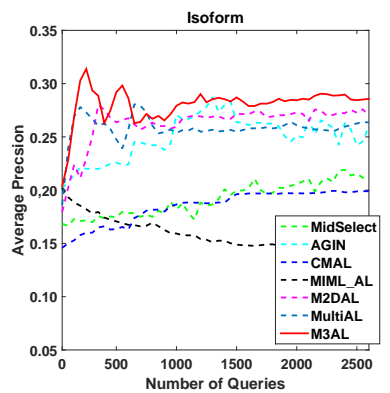

(h) Isoform

Fig. 2. Average Precision of comparing methods and M3AL on different datasets.

based bag-label uncertainty quantification of MIML-AL, and the selection of bag-label pairs. We empirically found that the performance of MIML-AL starts to increase after querying 3000 bag-label pairs, and reaches the peak after 10000 queries. (ii) Multi-instance vs. Single-instance: M2DAL, MultiAL and M3AL all focus on multi-view multi-label data, while M2DAL and MultiAL simple model each object as a single-instance. M3AL manifests a better performance than these two MVML active learning approaches across diverse datasets, especially the MVML datasets. This fact reflects the advantage of M3AL for querying complex M3 objects, and the effectiveness of M3AL on capturing the instance distributions for bag-label pairs selection. Besides, we also observe that all of these multiview based solutions can reach the peak faster than single-view counterparts. This observation again shows the significance of leveraging heterogeneous views of bags to reduce the labeling costs of M3 objects. Both M2DAL and M3AL use the decision hyper-planes, the commonality and individuality information to quantify the uncertainty of objects, but M2DAL is outperformed by M3AL. That is because M3AL additionally leverages the label correlations and instance distributions, to define a more informative bag-label pair selection criterion. M3AL considers the multiplicity of active learning on M3 data, so it achieves a better performance than these MIML-based and MVML-based active learning methods on the Isoform dataset. In addition, we find that multi-view algorithms (M2DAL, MultiAL and $\mathrm{M} 3 \mathrm{AL}$ ) on the real Isoform dataset show large fluctuations in the early queries. The possible reason is that the bag features of this dataset in multiple views are too sparse to capture data distribution. With the increase of labeled data, such situation is alleviated. In contrast, MIML-based methods directly use the concatenated bag features, thus they acquire a relatively stable performance.

(iii) Object-label pair query vs. Object query: Unlike other compared methods that select object-label pair for query, AGIN selects the most uncertain object for query. We find AGIN loses to compared methods in many cases. This confirms the advantage of object-label query than object query for multi-label active learning. Since AGIN considers the instance distribution of complex objects, it can still obtain a better performance than some of these compared methods.

To clearly present the results, Fig. 2 only plots the average results of ten independent rounds. We additionally report average results and standard deviation of ten independent rounds in Table S2 of the Supplementary file. We observe that these compared methods all have a small variance and M3AL clearly performs better than other compared methods. In addition, we additionally study the performance of M3AL against these compared methods under a fixed number of queries, and report the win/tie/loss ratios in Table S3 of the Supplementary file. M3AL dominantly achieves a better (or comparable) performance than any of these compared methods under a fixed number of queries. Overall, these results not only prove the effectiveness of M3AL on learning from single-view MIML and single-instance MVML datasets, but also on the more complex M3 dataset. In addition, these comparisons also suggest the necessity of M3AL to leverage the commonality, individuality and the instance distributions of bags across views.

\section{Ablation Study of $M 3 A L$}

We introduce four variants of M3AL for a comprehensive ablation study to further investigate the contribution factors of M3AL. These four variants are:

(i) $\mathbf{M 3 A L}(\mathbf{P})$ only considers the individuality $\left(\left\{\mathbf{P}^{v}\right\}_{v=1}^{V}\right)$ to 
measure the uncertainty of bag-label pairs.

(ii) M3AL(S) only uses the shared information of bags (S) for measuring the uncertainty of bag-label pairs.

(iii) $\mathbf{M} 3 \mathbf{A L}(\mathbf{n D B})$ disregards the instance distributions $D B(i)$ of bags across different views, and only uses the commonality and individuality to select bag-label pairs.

(iv) M3AL(nLC) does not use the label correlations (i.e., $\mathbf{W}(k, c))$ for selecting bag-label pairs.

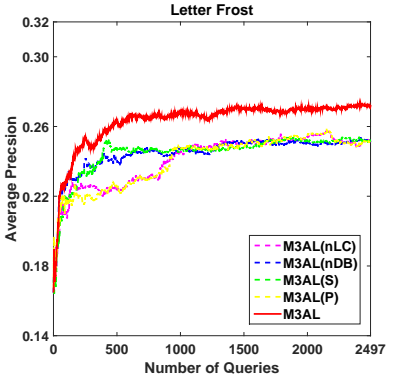

(a) Letter Frost

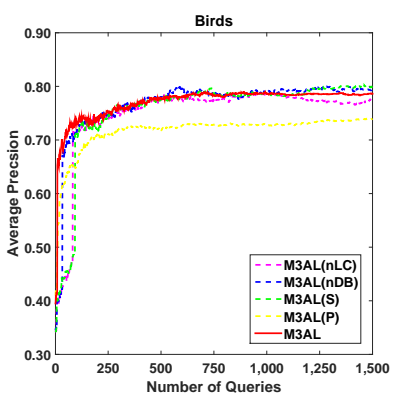

(c) Birds

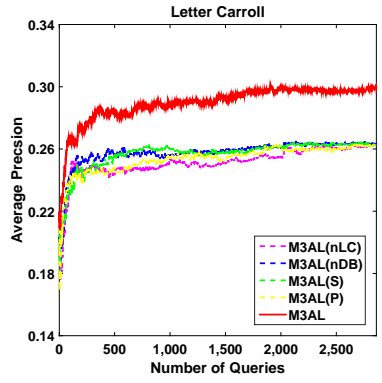

(b) Letter Carroll

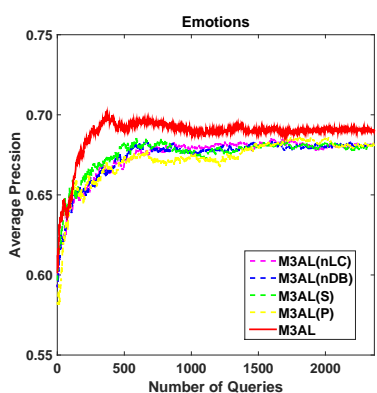

(d) Emotions
Fig. 3. Average Precision of M3AL and its variants on Letter Frost, Letter Carroll, Birds and Emotions datasets.

Fig. 3(a) 3(d) show the performance of these variants on Letter Frost, Letter Carroll, Birds and Emotions datasets, respectively. The experimental setting is fixed the same as the previous subsection. From Fig. 3, we have the following observations:

(i) The label correlations help to select informative bag-label pairs and thus boost the performance of multi-label active learning. This statement is confirmed by the performance margin between M3AL and its variant M3AL(nLC), which ignores the label correlations of multi-label bags. In addition, we observe the margin is more obvious as the number of queries increases, that is because label correlations become more reliable as more objects queried.

(ii) Leveraging the commonality and individuality of multiview data can also improve the performance of active learning on multi-view data. This observation is supported by the fact that M3AL obtains a better performance than M3AL(P) and $\mathrm{M} 3 \mathrm{AL}(\mathrm{S})$, which separately exploit the individual and shared information of multi-view data for selecting bag-label pair for query. We find that $\operatorname{M3AL}(\mathrm{S})$ achieves a better performance than M3AL(P), and that is because M3AL(S) fuses the information from different data views, while M3AL(P) does not have such a fusion, it judges a bag-label pair based on the insufficient information of individual views only. (iii) The instance distributions of bags within/across views contribute to the selection of informative bags. This observation can be clearly verified from the performance margin between M3AL and M3AL(nDB), which does not consider the instance distributions of bags.

Overall, these results not only prove the necessity to model individual and shared information of multi-view data, the label correlations of multi-label bags, and the instance distributions of bags within/across views to handle the multiplicity of active learning on M3 data, but also confirm the effectiveness of M3AL on modeling these factors for effective active learning on M3 data.

Due to the space limitation, the parameter sensitivity analysis is given in the supplementary file.

\section{CONCLUSION AND FUture WORK}

This paper studies how to effectively perform active learning on multi-view multi-instance multi-label objects, which is a practical, challenging, important but largely untouched topic. The proposed M3AL firstly learns the shared and individual information of bags across multiple views. Next, it designs a new query strategy combining the shared and individual information, and the instance distributions of bags to select the most informative bag-label pairs across multiple views for query. Experimental studies show the effectiveness of M3AL on learning from M3 objects with reduced query cost while maintaining a better performance. In the future, we will study more effective query strategies for selecting the informative bag-label (instance-label) pairs across multiple views, and mining nonlinear correlations between views by deep neural networks. The codes and datasets of M3AL are shared at mlda.swu.edu.cn/codes.php?name=M3AL

\section{REFERENCES}

[1] C. T. Nguyen, D. C. Zhan, and Z. H. Zhou, "Multi-modal image annotation with multi-instance multi-label lda," in IJCAI, 2013, pp. 15581564.

[2] Y. Yang, Y.-F. Wu, D.-C. Zhan, Z.-B. Liu, and Y. Jiang, "Complex object classification: A multi-modal multi-instance multi-label deep network with optimal transport," in KDD, 2018, pp. 2594-2603.

[3] Y. Xing, G. Yu, C. Domeniconi, J. Wang, Z. Zhang, and M. Guo, "Multiview multi-instance multi-label learning based on collaborative matrix factorization," in AAAI, 2019, pp. 5508-5515.

[4] Y. Yang, Z.-Y. Fu, D.-C. Zhan, Z.-B. Liu, and Y. Jiang, "Semisupervised multi-modal multi-instance multi-label deep network with optimal transport," TKDE, vol. 99, no. 1, pp. 1-13, 2020.

[5] T. Luo, W. Zhang, S. Qiu, Y. Yang, D. Yi, G. Wang, J. Ye, and J. Wang, "Functional annotation of human protein coding isoforms via non-convex multi-instance learning," in KDD, 2017, pp. 345-354.

[6] G. Yu, K. Wang, C. Domeniconi, M. Guo, and J. Wang, "Isoform function prediction based on bi-random walks on a heterogeneous network," Bioinformatics, vol. 36, no. 1, pp. 303-310, 2020.

[7] K. Wang, J. Wang, C. Domeniconi, X. Zhang, and G. Yu, "Differentiating isoform functions with collaborative matrix factorization," Bioinformatics, vol. 36, no. 6, pp. 1864-1871, 2020.

[8] C. T. Nguyen, X. Wang, J. Liu, and Z. H. Zhou, "Labeling complicated objects: multi-view multi-instance multi-label learning," in $A A A I, 2014$, pp. 2013-2019.

[9] B. Li, C. Yuan, W. Xiong, W. Hu, H. Peng, X. Ding, and S. Maybank, "Multi-view multi-instance learning based on joint sparse representation and multi-view dictionary learning." TPAMI, vol. 39, no. 12, pp. 2554 2560, 2017.

[10] B. Settles, M. Craven, and S. Ray, "Multiple-instance active learning," in NeurIPS, 2008, pp. 1289-1296. 
[11] G. Yu, J. Tu, J. Wang, C. Domeniconi, and X. Zhang, "Active multilabel crowd consensus," TNNLS, vol. 99, no. 1, pp. 1-12, 2020.

[12] S.-J. Huang, N. Gao, and S. Chen, "Multi-instance multi-label active learning," in IJCAI, 2017, pp. 1886-1892.

[13] R. Retz and F. Schwenker, "Active multi-instance multi-label learning," in Analysis of Large and Complex Data, 2016, pp. 91-101.

[14] X. Chen, G. Yu, C. Domeniconi, J. Wang, Z. Li, and Z. Zhang, "Cost effective multi-label active learning via querying subexamples," in ICDM 2018, pp. 905-910.

[15] M.-A. Carbonneau, E. Granger, and G. Gagnon, "Bag-level aggregation for multiple-instance active learning in instance classification problems," $T N N L S$, vol. 30, no. 5, pp. 1441-1451, 2019.

[16] G.-J. Qi, X.-S. Hua, Y. Rui, J. Tang, and H.-J. Zhang, "Two-dimensional active learning for image classification," in CVPR, 2008, pp. 1-8.

[17] S.-J. Huang, S. Chen, and Z.-H. Zhou, "Multi-label active learning: query type matters," in IJCAI, 2015, pp. 946-952.

[18] S. Luo, C. Zhang, W. Zhang, and X. Cao, "Consistent and specific multi-view subspace clustering," in AAAI, 2018, pp. 3730-3737.

[19] X. Zhang, J. Cheng, C. Xu, H. Lu, and S. Ma, "Multi-view multi-label active learning for image classification," in ICME, 2009, pp. 258-261.

[20] Z. Zhang, X. Jin, L. Li, G. Ding, and Q. Yang, "Multi-domain active learning for recommendation," in AAAI, 2016, pp. 2358-2364.

[21] B. Settles, "Active learning literature survey," University of WisconsinMadison Department of Computer Sciences, Tech. Rep., 2009.

[22] Y. Fu, X. Zhu, and B. Li, "A survey on instance selection for active learning," KAIS, vol. 35, no. 2, pp. 249-283, 2013.

[23] B. Yang, J.-T. Sun, T. Wang, and Z. Chen, "Effective multi-label active learning for text classification," in $K D D, 2009$, pp. 917-926.

[24] X. Li and Y. Guo, "Active learning with multi-label svm classification," in IJCAI, 2013, pp. 1479-1485.

[25] O. Reyes, C. Morell, and S. Ventura, "Effective active learning strategy for multi-label learning," Neurocomputing, vol. 273, pp. 494-508, 2018

[26] Z.-H. Zhou and M.-L. Zhang, "Multi-instance multi-label learning with application to scene classification," in NeurIPS, 2007, pp. 1609-1616.

[27] G. Yu, X. Chen, C. Domeniconi, J. Wang, Z. Li, Z. Zhang, and $\mathrm{X}$. Zhang, "Cmal: Cost-effective multi-label active learning by querying subexamples," TKDE, vol. 99, pp. 1-12, 2020.

[28] W. Wang and Z.-H. Zhou, "On multi-view active learning and the combination with semi-supervised learning," in ICML, 2008, pp. 1152 1159.

[29] J. Liu, Y. Jiang, Z. Li, Z.-H. Zhou, and H. Lu, "Partially shared latent factor learning with multiview data," $T N N L S$, vol. 26, no. 6, pp. 1233 1246, 2014.

[30] C. Sagonas, E. Ververas, Y. Panagakis, and S. Zafeiriou, "Recovering joint and individual components in facial data," TPAMI, vol. 40, no. 11, pp. 2668-2681, 2017.

[31] J. Hu, J. Lu, and Y.-P. Tan, "Sharable and individual multi-view metric learning," TPAMI, vol. 40, no. 9, pp. 2281-2288, 2017.

[32] S. Yao, G. Yu, J. Wang, C. Domeniconi, and X. Zhang, "Multi-view multiple clustering," in IJCAI, 2019, pp. 4121-4127.

[33] Q. Tan, G. Yu, J. Wang, C. Domeniconi, and X. Zhang, "Individualityand commonality-based multiview multilabel learning," IEEE T CYB, vol. 99, no. 1, pp. 1-12, 2020.

[34] H. Gao, F. Nie, X. Li, and H. Huang, "Multi-view subspace clustering," in ICCV, 2015, pp. 4238-4246.

[35] Z.-H. Zhou and M.-L. Zhang, "Solving multi-instance problems with classifier ensemble based on constructive clustering," $K A I S$, vol. 11, no. 2 , pp. 155-170, 2007.

[36] A. Gretton, O. Bousquet, A. Smola, and B. Schölkopf, "Measuring statistical dependence with hilbert-schmidt norms," in $A L T, 2005$, pp 63-77.

[37] Z. Zhou, M. Zhang, S. Huang, and Y. Li, "Multi-instance multi-label learning," Artificial Intelligence, vol. 176, no. 1, pp. 2291-2320, 2012.

[38] F. Hausdorff, Set theory. American Mathematical Society, 1991.

[39] M. Belkin, P. Niyogi, and V. Sindhwani, "Manifold regularization: A geometric framework for learning from labeled and unlabeled examples," $J M L R$, vol. 7, no. 11, pp. 2399-2434, 2006.

[40] M.-L. Zhang and K. Zhang, "Multi-label learning by exploiting label dependency," in KDD, 2010, pp. 999-1008.

[41] C.-C. Chang and C.-J. Lin, "Libsvm: A library for support vector machines," TIST, vol. 2, no. 3, pp. 1-27, 2011.

[42] D. S. Watkins, Fundamentals of matrix computations. John Wiley \& Sons, 2004.

[43] J. Winn, A. Criminisi, and T. Minka, "Object categorization by learned universal visual dictionary," in ICCV, 2005, pp. 1800-1807.

[44] F. Briggs, X. Z. Fern, and R. Raich, "Rank-loss support instance machines for miml instance annotation," in $K D D, 2012$, pp. 534-542.
[45] M. Guillaumin, J. Verbeek, and C. Schmid, "Multimodal semi-supervised learning for image classification," in $C V P R, 2010$, pp. 902-909.

[46] E. L. Gibaja, J. M. Moyano, and S. Ventura, "An ensemble-based approach for multi-view multi-label classification," Progress in Artificial Intelligence, vol. 5, no. 4, pp. 251-259, 2016.

[47] Y. Li, J. Hu, y. Jiang, and Z. Zhou, "Two-dimensional active learning for image classification," in AAAI, 2012, pp. 1012-1018.

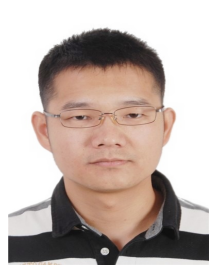

Guoxian Yu is a Professor at the School of Software, Shandong University, Jinan, China. He received the $\mathrm{PhD}$ in Computer Science from South China University of Technology, Guangzhou, China in 2013 His current research interests include data mining and bioinformatics. He severs as reviewers for ICML, KDD, ICDM, IJCAI, TKDE, TNNLS, Bioinformatics and other prestigious conferences and journals.

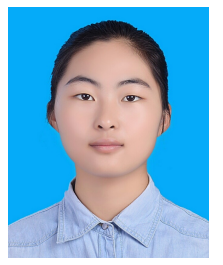

Yuying Xing received the MPhil degree from the College of Computer and Information Sciences, Southwest University, Chongqing, China in 2020. She is currently an algorithm engineer with the Alibaba Group, specializing in multi-view data analysis, network embedding and anomaly detection.

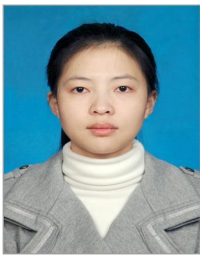

Jun Wang is a Professor with the Joint SDU-NTU Centre for Artificial Intelligence Research, Shandong University. She received BSc degree in Computer Science, MEng degree in Computer Science and $\mathrm{PhD}$ in Artificial Intelligence from Harbin Institute of Technology, Harbin, China in 2004, 2006 and 2010, respectively. Her current research interests include data mining and their applications in bioinformatics.

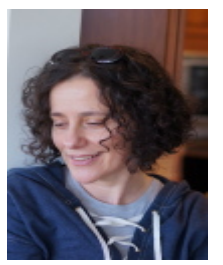

Carlotta Domeniconi is an Associate Professor in the Department of Computer Science at George Mason University. Her research interests include machine learning, data mining and bioinformatics. She serves as the Associate Editor of IEEE Transactions on Knowledge and Data Engineering, and Knowledge and Information Systems. She was an Associate Editor of IEEE Transactions on Neural Networks and Learning System.

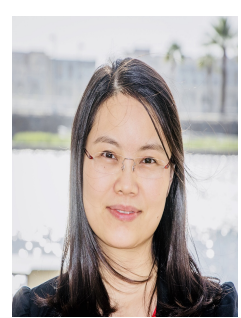

Xiangliang Zhang is an Associate Professor and directs the Machine Intelligence and Knowledge Engineering (MINE) Laboratory in King Abdullah University of Science and Technology (KAUST). She earned her $\mathrm{PhD}$ degree in Computer Science with great honors from INRIA-University Paris-Sud 11 , France, in 2010. Her main research interests and experiences are in diverse areas of machine learning and data mining. 